\title{
Equivalence of different descriptions for $\eta$ Particle in Simplest Little Higgs Model
}

\author{
Ran Lu, Qing Wang \\ Department of Physics, Tsinghua University,Beijing 100084,P.R.China* \\ Center for High Energy Physics, Tsinghua University, Beijing 100084, P.R.Chind
}

(Dated: November 8, 2018)

\begin{abstract}
In $S U(3)$ simplest little Higgs model, a characteristic particle is the light pseudoscalar boson $\eta$, it leads interesting signals in the LHC/ILC and is studied in literature with different parameterizations. In this work, we show that these different descriptions for $\eta$ particle are equivalent up to some $S U(3)$ rotations as long as we suitably redefine the pseudo Goldstone boson fields. We evaluate the necessary $S U(3)$ rotations and built up explicit expressions for redefined fields.
\end{abstract}

PACS numbers: 11.10.Lm, 11.30.Rd, 12.60.Fr, 14.80.Mz

\footnotetext{
* mailing address

${ }^{\dagger}$ Electronic address: luran@mails.tsinghua.edu.cn
} 
The Higgs boson is the last ingredient of the Standard model (SM), its properties attract lots of our attentions either in experiments and in theories. Experimentally, precision measurements of electroweak parameters give indirect limit on the $m_{H}$ to be less than $186 \mathrm{GeV}$ at $95 \%$ confidence level(C.L.) [1] and direct search by LEPII experiments gives a lower bound of $114.4 \mathrm{GeV}$ at the $95 \%$ C.L. [2]. Theoretically, numerous new physics models are invented with different roles of Higgs in the models. Among them, a class of little Higgs models are proposed to soften hierarchy problem [3]. In these models, relatively light Higgs boson mass is due to its identity as a pseudo Goldstone boson of some enlarged global symmetries. Among various little Higgs models, the simplest little Higgs model [4, 5] is attractive due to its relative simple theory structures. The model is based on the $\left[S U(3) \times U(1)_{X}\right]^{2}$ global symmetry with its diagonal subgroup $S U(3) \times U(1)_{X}$ gauged. The vacuum expectation values of two $S U(3)$ triplet scalar fields $\Phi_{1}$ and $\Phi_{2}$ spontaneously break both the global and the gauge symmetries. Uneaten pseudo Goldstone bosons are $S U(2)_{L}$ doublet $h$ and a pseudoscalar $\eta$. The typical feature of simplest little Higgs model is the existence of this $\eta$ particle, as a pseudo Goldstone boson, it should be light and the upper and lower bounds of its mass were estimated in Ref.[6], roughly speaking in $\mathrm{GeV}$ range below electroweak scale $v=250 \mathrm{GeV}$. Due to its lightness, $\eta$ may affects the physics significantly and leads interesting phenomenologies at next generation of high energy colliders [6, 7, 8, 9, 10]. For the theoretical computations on the phenomenologies related to $\eta$ particle, pseudo Goldstone bosons $h$ and $\eta$ should be written as a combination field $\Theta$ which is defined as

$$
\Theta=\frac{1}{f}\left[\frac{\eta}{\sqrt{2}} T+\Theta_{h}\right] \quad \Theta_{h}=\left(\begin{array}{cc}
0 & h \\
h^{\dagger} & 0
\end{array}\right)
$$

$h$ is a doublet which will become the SM Higgs, $\eta$ is a real pseudoscalar field, $T$ is its generator matrix, the original choice of $T$ matrix is $T=T_{8} \sqrt{3} / \sqrt{2}[4]$ with $T_{8}=\operatorname{diag}(1,1,-2) / 2 \sqrt{3}$ be $S U(3)$ generator. The two $S U(3)$ triplet scalar fields in the model are expressed in terms of pseudo Goldstone boson field $\Theta$ and their vacuum expectation values $f_{1}$ and $f_{2}$ as [5]

$$
\Phi_{1}=e^{i \Theta \frac{f_{2}}{f_{1}}}\left(\begin{array}{c}
0 \\
0 \\
f_{1}
\end{array}\right) \quad \Phi_{2}=e^{-i \Theta \frac{f_{1}}{f_{2}}}\left(\begin{array}{c}
0 \\
0 \\
f_{2}
\end{array}\right)
$$

where $f^{2}=f_{1}^{2}+f_{2}^{2}$, With them the bosonic part Lagrangian of the model involving pseudo Goldstone field is 


$$
\mathcal{L}=\left[\left(\partial_{\mu}+i g A_{\mu}^{a} T^{a}-\frac{i}{3} g_{x} A_{\mu}^{x}\right) \Phi_{i}\right]^{\dagger}\left[\left(\partial_{\mu}+i g A_{\mu}^{a} T^{a}-\frac{i}{3} g_{x} A_{\mu}^{x}\right) \Phi_{i}\right]+\mu^{2}\left(\Phi_{1}^{\dagger} \Phi_{2}+\Phi_{2}^{\dagger} \Phi_{1}\right)
$$

with $A_{\mu}^{a}$ and $A_{\mu}^{x}$ be $S U(3)$ and $U(1)_{X}$ gauge fields respectively.

In practical calculations, for pseudo Goldstone field $\Theta$ given in (1), people use unit matrix $I$ to replace original $S U(3)$ generator $T=T_{8} \sqrt{3} / \sqrt{2}[5,6$, 7, 8, 10]. The reason to replace $S U$ (3) generator with unit matrix is explained in Ref. [6] in a footnote as that the choice of $S U$ (3) generator introduce kinetic mixing of the $\eta$ with unphysical Goldstone bosons, removing this mixing by appropriate field redefinitions is equivalent to choosing $T$ proportional to the unit matrix. This explanation is not easy to read out directly from (2) and (3). We have checked that the global symmetry broken is $\mathrm{SO}(6) / \mathrm{SO}(5)$, both $T_{8}$ and $I$ can be embedded into $\mathrm{SO}(6)$ and they are all belong to a same broken generator plus some other different unbroken generators, in this sense, two parameterizations should be identical. I commute with all the other broken generators and the mixing should be minimal. If this judgment is correct, $T_{8}$ must produce same result as that from unit matrix $I$. Consider the importance of $\eta$ particle in the simplest little Higgs model, in this paper, we present a explicit proof of this equivalence by showing that we can choose redefined fields $\tilde{\eta}$ and $\tilde{h}$ to make the changes of two $S U(3)$ triplet fields $\Phi_{1}$ and $\Phi_{2}$ from choice of $T=T_{8} \sqrt{3} / \sqrt{2}$ to $T=I$ is equal to a local $S U(3)$ rotation and therefore can be rotated away. i.e.

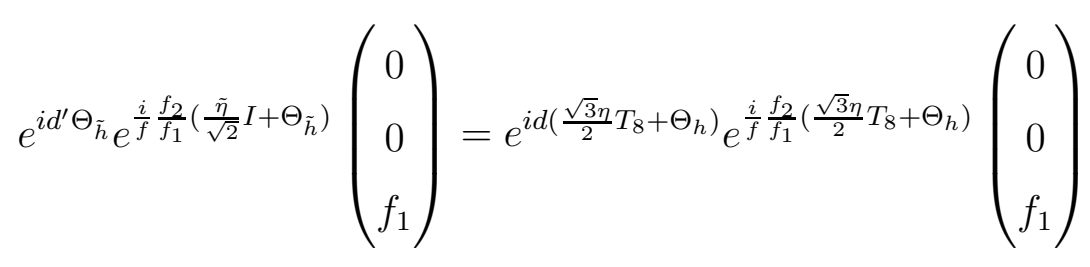

and

$$
e^{i d^{\prime} \Theta_{\tilde{h}}} e^{-\frac{i}{f} \frac{f_{1}}{f_{2}}\left(\frac{\tilde{n}}{\sqrt{2}} I+\Theta_{\tilde{h}}\right)}\left(\begin{array}{c}
0 \\
0 \\
f_{2}
\end{array}\right)=e^{i d\left(\frac{\sqrt{3} \eta}{2} T_{8}+\Theta_{h}\right)} e^{-\frac{i}{f} \frac{f_{1}}{f_{2}}\left(\frac{\sqrt{3} \eta}{2} T_{8}+\Theta_{h}\right)}\left(\begin{array}{c}
0 \\
0 \\
f_{2}
\end{array}\right)
$$

where $d$ and $d^{\prime}$ are two $\eta$ and $h$ dependent dimensional parameters which make the total argument on the exponential dimensionless, we will fix $d$ and $d^{\prime}$ later. Once (4) and (5) are valid, we can take $S U(3)$ rotations to rotate away the phase factor $e^{i d^{\prime} \Theta_{\tilde{h}}}$ in l.h.s. and 
$e^{i d\left(\frac{\sqrt{3} \eta}{2} T_{8}+\Theta_{h}\right)}$ in r.h.s. of (44) and (55), then choice of $T=T_{8} \sqrt{3} / \sqrt{2}$ and $T=I$ become equivalent. To prove (4) and (5), we find they are equivalent to following identity

$$
\begin{gathered}
e^{i \frac{f_{2}}{f f_{1}} \frac{\tilde{\eta}}{\sqrt{2}} I+i\left(d^{\prime}+\frac{f_{2}}{f f_{1}}\right) \Theta_{\tilde{h}}} \Phi_{0}=e^{i\left(d+\frac{f_{2}}{f f_{1}}\right)\left(\frac{\sqrt{3} \eta}{2} T_{8}+\Theta_{h}\right)} \Phi_{0} \\
e^{-i \frac{f_{1}}{f f_{2}} \frac{\tilde{n}}{\sqrt{2}} I+i\left(d^{\prime}-\frac{f_{1}}{f f_{2}}\right) \Theta_{\tilde{h}}} \Phi_{0}=e^{i\left(d-\frac{f_{1}}{f f_{2}}\right)\left(\frac{\sqrt{3} \eta}{2} T_{8}+\Theta_{h}\right)} \Phi_{0}
\end{gathered}
$$

with $\Phi_{0}=(0,0,1)^{T}$. We now discuss how to realize (6) and (7). Because the special form the parametrization, the exponential of $e^{i \Theta_{\tilde{h}}}$ at l.h.s. of (6) and (7) can be work out explicitly through explicit computations order by orders, one can show that for some constant $c$

$$
e^{i c \Theta_{\tilde{h}}}=I+\frac{[\cos (c \tilde{H})-1]}{\tilde{H}^{2}} B_{\tilde{h}}+i \frac{\sin (c \tilde{H})}{\tilde{H}} \Theta_{\tilde{h}}
$$

with $B_{\tilde{h}}=\Theta_{\tilde{h}}^{2}=\left(\begin{array}{cc}\tilde{h} \tilde{h}^{\dagger} & 0 \\ 0 & \tilde{H}^{2}\end{array}\right)$, and $\tilde{H}=\left(\tilde{h}^{\dagger} \tilde{h}\right)^{\frac{1}{2}}$, Eq.(8) then leads

$$
e^{i\left(\frac{\mathrm{c}^{\prime} \tilde{\tilde{n}}}{\sqrt{2}} I+c \Theta_{\tilde{h}}\right)} \Phi_{0}=e^{i c^{\prime} \frac{\tilde{n}}{\sqrt{2}}}\left[\cos (c \tilde{H}) \Phi_{0}+i \frac{\sin (c \tilde{H})}{\tilde{H}} \Phi_{\tilde{h}}\right]
$$

with definition of $\Phi_{\tilde{h}}=\left(\begin{array}{l}\tilde{h} \\ 0\end{array}\right)$.

Further calculations on $e^{i c\left(\frac{\sqrt{3} \eta}{2} T_{8}+\Theta_{h}\right)}$ needs some care, since $T_{8}$ matrix is not commute with $\Theta_{h}$. Consider

$$
\begin{aligned}
& T_{8}=\frac{1}{2 \sqrt{3}}\left(\begin{array}{llc}
1 & 0 & 0 \\
0 & 1 & 0 \\
0 & 0 & -2
\end{array}\right)=\frac{1}{2 \sqrt{3}}(I-3 \tilde{T}) \\
& \tilde{T}=\left(\begin{array}{lll}
0 & 0 & 0 \\
0 & 0 & 0 \\
0 & 0 & 1
\end{array}\right)
\end{aligned}
$$

then we consider

$$
e^{i c\left(\frac{\sqrt{3} \eta}{2} T_{8}+\Theta_{h}\right)} \Phi_{0}=e^{i \frac{c \eta}{4}} e^{i c\left(-\frac{3 \eta}{4} \tilde{T}+\Theta_{h}\right)} \Phi_{0}
$$

One can check that

$$
\left(-\frac{3 \eta}{4} \tilde{T}+\Theta_{h}\right)^{n}=a_{n-1}\left(-\frac{3 \eta}{4} \tilde{T}+\Theta_{h}\right)+a_{n-2} B_{h}
$$


with $a_{n}=(-3 \eta / 4) a_{n-1}+H^{2} a_{n-2}, n \geq 2, a_{0}=1, a_{1}=-3 \eta / 4, H=\left(h^{\dagger} h\right)^{\frac{1}{2}}$. Solve the recursion relation, we obtain

$$
a_{n}=\frac{\alpha^{n+1}-\beta^{n+1}}{\alpha-\beta}
$$

where

$$
\begin{aligned}
\alpha & =\frac{-3 \eta / 4+\rho}{2}, \quad \beta=\frac{-3 \eta / 4-\rho}{2} \\
\rho & =\sqrt{9 \eta^{2} / 16+4 H^{2}}
\end{aligned}
$$

With this result, then

$e^{i c\left(-\frac{3 \eta}{4} \tilde{T}+\Theta_{h}\right)}=I+\left[-1+e^{-\frac{3 i c \eta}{8}}\left(-\frac{3 i \eta / 4}{\rho} \sin \frac{c \rho}{2}+\cos \frac{c \rho}{2}\right)\right] \frac{B_{h}}{H^{2}}+e^{-\frac{3 i c \eta}{8}} \frac{2 i \sin \frac{c \rho}{2}}{\rho}\left(-\frac{3 \eta}{4} \tilde{T}+\Theta_{h}\right.$

and

$$
e^{i c\left(-\frac{3 \eta}{4} \tilde{T}+\Theta_{h}\right)} \Phi_{0}=e^{-\frac{3 i c \eta}{8}}\left[-\frac{i 3 \eta / 4}{\rho} \sin \frac{c \rho}{2}+\cos \frac{c \rho}{2}\right] \Phi_{0}-e^{-\frac{3 i c \eta}{8}} \frac{2 i \sin \frac{c \rho}{2}}{\rho} \Phi_{h}
$$

Introduce a phase angle

$$
\tan \Delta(x, y)=\frac{3 x / 4}{\sqrt{9 x^{2} / 16+4 y^{2}}} \tan \frac{\sqrt{9 x^{2} / 16+4 y^{2}}}{2}
$$

$e^{i c\left(\frac{\sqrt{3} \eta}{2} T_{8}+\Theta_{h}\right)} \Phi_{0}$ then can be expressed as

$$
e^{i c\left(\frac{\sqrt{3} \eta}{2} T_{8}+\Theta_{h}\right)} \Phi_{0}=e^{-i\left\{\frac{c \eta}{8}+\Delta[c \eta, c H]\right\}} \sqrt{1-\frac{4 H^{2}}{\rho^{2}} \sin ^{2} \frac{c \rho}{2}} \Phi_{0}+e^{-\frac{i c \eta}{8} \frac{2 i \sin \frac{c \rho}{2}}{\rho}} \Phi_{h}
$$

Demand $\eta$ and $h$ fields satisfy

$$
\eta=\frac{1}{a} \tilde{\eta} \quad h=\frac{1}{b} e^{-i \Delta[c \eta, c H]} \tilde{h}
$$

with $a$ and $b$ be two dimensionless parameters depend on $\eta$ and $h$, then compare (19) with (9), as long as we demand

$$
\begin{aligned}
& b_{i}^{\prime} a \frac{\eta}{\sqrt{2}}+\delta=-\frac{c_{i} \eta}{8}-\Delta\left[c_{i} \eta, c_{i} H\right] \\
& \left|\cos \left(c_{i}^{\prime} b H\right)\right|=\sqrt{1-\frac{4 H^{2}}{\rho^{2}} \sin ^{2} \frac{c_{i} \rho}{2}} \\
& \frac{\sin \left(c_{i}^{\prime} b H\right)}{H}=\frac{2 \sin \frac{c_{i} \rho}{2}}{\rho}
\end{aligned}
$$


with $i=1,2, \delta=\pi, 0$ for $\cos \left(c_{i}^{\prime} b H\right)$ take negative and positive values respectively, and

$$
\begin{array}{lll}
c_{1}=d+b_{1} & c_{1}^{\prime}=d^{\prime}+b_{1} & b_{1}=\frac{f_{2}}{f f_{1}} \\
c_{2}=d-b_{2} & c_{2}^{\prime}=d^{\prime}-b_{2} & b_{2}=\frac{f_{1}}{f f_{2}}
\end{array}
$$

then we will have (61) and (77). It is easy to see (221) and (23) are equivalent, then only (21) and (23) are independent equations from which and (24) we can solve out parameters $a, b, d, d^{\prime}$. With the solution and combine with (18), we can rewrite (20) as

$$
\begin{aligned}
& \tilde{\eta}=-\frac{c_{1} \eta}{4 b_{1}^{\prime} \sqrt{2}}-\frac{\sqrt{2}}{b_{1}^{\prime}} \arctan \left[\frac{3 \eta / 4}{\rho} \tan \frac{c_{1} \rho}{2}\right] \\
& \tilde{h}=\frac{h e^{i \Delta\left[c_{1} \eta, c_{1} H\right]}}{c_{1}^{\prime} H} \arcsin \left[\frac{2 H \sin \frac{c_{1} \rho}{2}}{\rho}\right]
\end{aligned}
$$

in which $c_{1}$ and $c_{1}^{\prime}$ are constrained by

$$
\begin{aligned}
& \frac{c_{1} \eta}{4 b_{1}^{\prime} \sqrt{2}}+\frac{\sqrt{2}}{b_{1}^{\prime}} \arctan \left[\frac{3 \eta / 4}{\rho} \tan \frac{c_{1} \rho}{2}\right] \\
& =\frac{c_{2} \eta}{4 b_{2}^{\prime} \sqrt{2}}+\frac{\sqrt{2}}{b_{2}^{\prime}} \arctan \left[\frac{3 \eta / 4}{\rho} \tan \frac{c_{2} \rho}{2}\right] \\
& \frac{1}{c_{1}^{\prime}} e^{i \Delta\left[c_{1} \eta, c_{1} H\right]} \arcsin \left[\frac{2 H \sin \frac{c_{1} \rho}{2}}{\rho}\right] \\
& =\frac{1}{c_{2}^{\prime}} e^{i \Delta\left[c_{2} \eta, c_{2} H\right]} \arcsin \left[\frac{2 H \sin \frac{c_{2} \rho}{2}}{\rho}\right]
\end{aligned}
$$

in which from (24), $c_{2}$ and $c_{2}^{\prime}$ related to $c_{1}$ and $c_{1}^{\prime}$ by

$$
c_{2}=c_{1}-b_{1}-b_{2} \quad c_{2}^{\prime}=c_{1}^{\prime}-b_{1}-b_{2}
$$

Once $c_{1}$ and $c_{1}^{\prime}$ is fixed, parameters $d$ and $d^{\prime}$ are fixed then through (24). Up to this stage, we achieve the proof of (6) and (77) and then the choice of $T=T_{8} \sqrt{3} / \sqrt{2}$ and $T=I$ become equivalent. This method can be applied further to the case of that $T$ matrix is proportional to $\tilde{T}$ and then as predicted by Ref.[6] that this choice of $T$ matrix is also equivalent to $T=T_{8} \sqrt{3} / \sqrt{2}$ and $T=I$. 


\section{Acknowledgments}

This work was supported by National Science Foundation of China (NSFC) under Grant No. 10435040. We also thank Dr. David Rainwater for helpful discussions.

[1] W.-M.Yao et al. (Particle Data Group), J. Phys. G33,1(2006)

[2] R.Barate et al., Phys. Lett. B565, 61(2003)

[3] N.Arkani-Hamed, A.G.Cohen and H.Georgi, Phys. Lett. B513,232(2001);

N.Arkani-Hamed, A.G.Cohen, E.Katz,A.E.Nelson, T.Gregoire and J.G.Wacker, JHEP 0208, $021(2002)$

[4] D.E.Kaplan, M.Schmaltz, JHEP 0310,039(2003)

[5] M.Schmaltz, JHEP 0408, 056(2004)

[6] W.Kilian, D.Rainwater and J.Reuter, Phys. Rev. D71,015008(2005)

[7] W. Kilian, J.Reuter, D. Rainwater, hep-ph/0507081

[8] K. Cheung, J.Song, hep-ph/0611294

[9] A.Abada, G.Bhattacharyya, M.Losada, Phys.Rev. D73, 033006(2006)

[10] J.Lee, hep-ph/0504136 